\title{
Tunable and Efficient Long Range Energy Transfer Via Graphene Plasmon Modes
}

\author{
$\underline{\text { Vasileios D. Karanikolas }}^{1}$, Cristian A. Marocico' ${ }^{1}$ and A. Louise Bradley ${ }^{1}$ \\ ${ }^{1}$ Photonics Group, School of Physics and CRANN, Trinity College Dublin, College Green 2, Dublin, Ireland \\ e-mail: karanikv@tcd.ie
}

\begin{abstract}
We present a theoretical investigation of the energy transfer efficiency between quantum systems placed in the vicinity of a doped graphene layer using a Green's tensor formalism. The direct interaction, Förster mechanism, between donor and acceptor dipoles dominates when they are close to each other, but is modified from its free-space value due to the presence of the graphene monolayer. In particular, the Förster radius, $R_{0}$, is modified from its free space value of $R_{0}=19 \mathrm{~nm}$ and can reach values of $100 \mathrm{~nm}$. As the donor-acceptor distance is increased the direct interaction is overshadowed by the interaction via the propagating graphene plasmon mode. Due to the large propagation length of the surface plasmon mode on graphene, energy transfer efficiencies as high as $\mathbf{5 0 \%}$ can still be achieved for distances as large as 300 $\mathrm{nm}$. The interaction via the surface plasmon mode is tunable via the doping of the graphene monolayer and the surface plasmon channel can also be switched-off this way.
\end{abstract}

\section{INTRODUCTION}

Surface plasmon polariton (SPP) modes can be used to confine light to sub-wavelength structures, an effect that is advantageous for various applications in biosensing devices, light harvesting, optical nanoantennas and quantum information processing [1]. Surface plasmon polaritons are collective oscillations of electrons and the electromagnetic field that are excited at the interface between a dielectric and a conductor and can propagate along that interface. Noble metals are routinely used as the conducting medium, although they suffer from large Ohmic losses. Graphene constitutes an alternative to using noble metals for plasmonic applications due to the fact that it exhibit lower material losses [2]. Furthermore, the optical properties of this 2D atomically thick monolayer can be tuned through gating.

In this contribution we theoretically investigate the energy transfer (ET) efficiency between a pair of quantum emitters (QEs) which are placed in the vicinity of a free standing graphene monolayer (GM). An excited donor QE has two relaxation paths, either through spontaneous emission (SE) to the GM or by energy transfer (ET) to an acceptor QE. The ET efficiency reflects the competiton between the two processes. In our model, taking into account the SE rate modified by the presence of the GM, we can report energy transfer efficiencies above $50 \%$, over distances of hundreds of nanometers along the GM, thus opening opportunities for experimental investigations of the coupled QE-graphene nanostructure. We use quantum emitters with optical properties corresponding to real physical systems. The ET efficiency can be tuned, through gating of the GM, with potential for applications such as switching and sensing devices, light harvesting, plasmonic rulers and quantum computing.

The paper is structured as follow in Sec. II we give a brief introduction to the optical properties of the GM and the main quantities used to define the ET efficiency. In Sec. III we give the main results of our theoretical analysis, in particular, the tuning of the ET efficiency for a given pair of QEs and the mechanisms dominating in e different distance regimes. Finally, in Sec. IV we give a summary of the results and conclusions drawn.

\section{GRAPHENE CONDUCTIVITY AND ENERGY TRANSFER RFFICIENCY}

The graphene in-plane conductivity, $\sigma$, is calculated in the random phase approximation. This quantity is mainly controlled by electron-hole pairs and can be divided into intraband and interband contributions, $\sigma=\sigma_{\text {intra }}+$ $\sigma_{\text {inter }}$. The $\sigma_{\text {intra }}$ term is described by a Drude type of model. Throughout this analysis we consider that the GM is at a temperature of $T=300^{\circ} \mathrm{K}$, with arelaxation time $\tau=1 \mathrm{ps}$ [3].

In this contribution we consider statistical ensembles of emitters, thus we will use an emission spectrum, $f_{D}(\lambda)$, to describe the donor QE. Then the spontaneous emission rate, $k_{S E}$ for the ensemble can be described as

$$
k_{S E} \propto \int_{0}^{\infty} d \lambda f_{D}(\lambda) \operatorname{Im}\left(\hat{\mu}_{\mathrm{D}} \cdot \mathbf{G}\left(\mathbf{r}_{\mathrm{D}}, \mathbf{r}_{\mathrm{D}}, \lambda\right) \cdot \hat{\mu}_{\mathrm{D}}\right)
$$



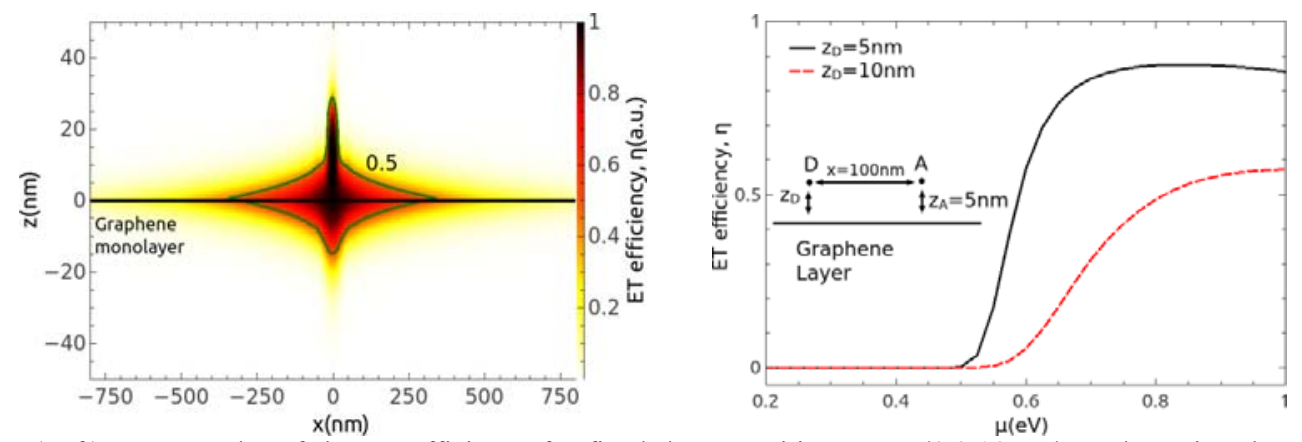

Fig. 1. (Left) Contour plot of the ET efficiency for fixed donor position, $r_{D}=(0,0,10 \mathrm{~nm})$, and varying the acceptor position at the $x z$-plane. (Right) The ET efficiency for fixed donor-acceptor separation, $100 \mathrm{~nm}$, and two donor positions, $z_{\mathrm{D}}$, above the GM.

where $\mathbf{r}_{\mathrm{D}}$ is the donor QE position [4].

When calculating the energy transfer rate, $k_{E T}$, we have the donor emission spectrum $f_{D}(\lambda)$ and acceptor absorption cross-section $\sigma_{A}(\lambda)[4]$,

$$
k_{E T} \propto k_{S E} \int_{0}^{\infty} \frac{d \lambda}{\lambda^{2}} f_{D}(\lambda)\left|\hat{\mu}_{D} \cdot \mathbf{G}\left(\mathbf{r}_{\mathrm{A}}, \mathbf{r}_{\mathrm{D}}, \lambda\right) \cdot \hat{\mu}_{A}\right|^{2} \sigma_{A}(\lambda)
$$

where $\mathbf{r}_{D}$ and $\mathbf{r}_{A}$ are the donor and acceptor positions, respectively. In Eq.(1) and Eq.(2) $\mathbf{G}\left(\mathbf{r}_{\mathrm{A}}, \mathbf{r}_{\mathrm{D}}, \lambda\right)$ is the Green's tensor for the particular geometry.

We now are now able to define the ET efficiency $\eta$ as

$$
\eta=\frac{k_{E T}}{k_{E T}+k_{S E}}
$$

This quantity gives the relative contribution of the ET process to the total decay rate of the donor. If $\eta>50 \%$ then relaxation of the donor occurs mainly by ET to the acceptor.

For the rest of the paper we consider donor emission and acceptor absorption spectra given by Gaussian distributions, which resemble real physical systems. The donor emission peak and acceptor absorption maximum coincide at $\lambda=2 \mu \mathrm{m}$. For this particular pair of QEs we have a free-space Förster radius of $R_{0}=$ $19 \mathrm{~nm}$, which is a characteristic distance at which the ET efficiency has a value $\eta=50 \%$.

\section{ENERGY TRANSFER EFFICIENCY}

In Fig. 1(left) we present a contour plot of the ET efficiency for the donor-acceptor pair, with spectral properties described above. The donor and acceptor positions are fixed at $r_{D}=(0,0,10 \mathrm{~nm})$, and $r_{A}=(x, 0, z)$, respectively. For a chemical potential $\mu=1.0 \mathrm{eV}$ the emission and absorption spectrum overlap strongly with the Green's tensor function, $\mathbf{G}\left(\mathbf{r}_{\mathrm{A}}, \mathbf{r}_{\mathrm{D}}, \lambda\right)$. For this case the ET efficiency, $\eta$, has values above $70 \%$ even for separations along the GM as large as $100 \mathrm{~nm}$, and the $50 \%$ efficiency distance is around $300 \mathrm{~nm}$ [5]. This value is large compared to the free-space Förster radius of $R_{0}=19 \mathrm{~nm}$. In Fig. 1(right) we fix the acceptor position at $r_{A}=(100 \mathrm{~nm}, 0,5 \mathrm{~nm})$ and consider two donor position, $r_{D}=(0,0,5 \mathrm{~nm})$ and $r_{D}=(0,0,10 \mathrm{~nm})$, while we vary the value of the chemical potential, $\mu$. We clearly observe the tunability of the ET efficiency for the given QEs pair, where for values of $\mu<0.6 \mathrm{eV}$ the ET rate channel is completely switched-off, due to the fact that the graphene plasmon (GP) mode is not excited under these conditions.

In Fig. 2(left) we present the ET rate, $k_{E T}$, as a function of the in-plane separation between the donor and acceptor, when their elevation above the GM is $z_{D}=z_{A}=10 \mathrm{~nm}$ on the same side of the GM. We have fitted the near-field with a Förster-type model, $\left(R_{0} / x\right)^{n}$, where $R_{0}$ is the Förster radius. At small separations the fit yields the values $n=6$ typical of Förster ET, but we see that the Förster radius is modified from the free-space value. The fact that at small separations, $x<20 \mathrm{~nm}$, the ET rate, $k_{E T}$, follows an $n=6$ dependence shows that the homogeneous part of the Green's tensor dominates, modified by the donor-acceptor interaction with the GM. Thus, there is an enhancement of the Förster radius, which depends on the value of the chemical potential $\mu$. When $\mu=1.0 \mathrm{eV}$ we have the largest value of the Förster radius, $R_{0}=94.3 \mathrm{~nm}$, due to our choice of donor and acceptor. For the off-resonance case, $\mu=0.4 \mathrm{eV}$, the Förster radius is $R_{0}=54.2 \mathrm{~nm}$. At larger 

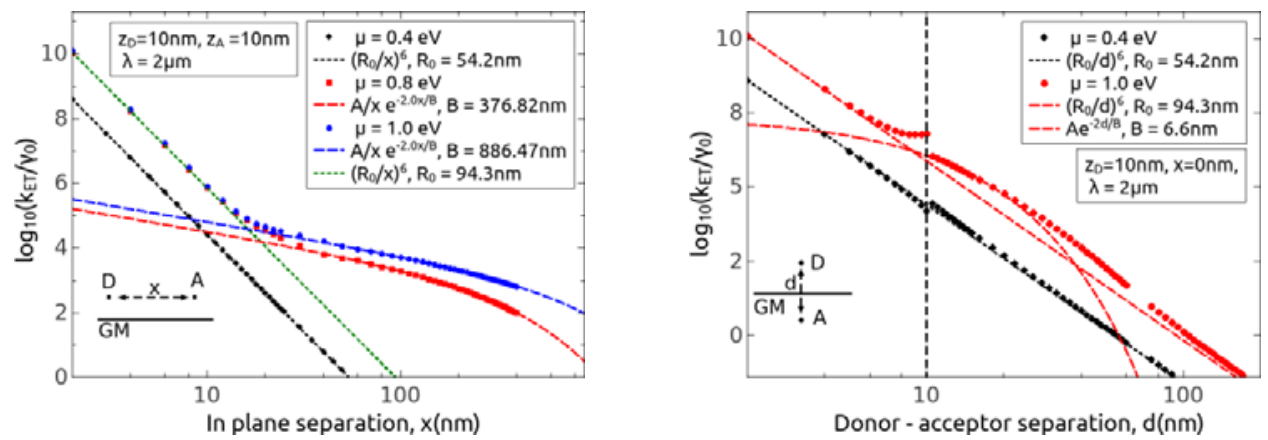

Fig. 2. ET rate, $k_{E T}$, for fixed donor position, $r_{D}=(0,0,10 \mathrm{~nm})$ and variable acceptor position, $r_{A}$, for different values of the chemical potential, $\mu$. (Left) Varying the in-plane separation of the acceptor $r_{A}=(x, 0,10 \mathrm{~nm})$ (Right) Varying the perpendicular acceptor position $r_{A}=\left(0,0, z_{A}\right)$, with $d=z_{D}-z_{A}$.

donor-acceptor separations, we use the expression $A / x \exp (-2 x / B)$ to fit the calculated ET rate which represents the dependence of the GP field intensity on the in-plane separation $\mathrm{x}$. The fitting parameter B is found to be in very good agreement with the GP propagation length extracted from the dispersion relation, $\omega\left(k_{s}\right)$, confirming that, away from the near-field, the interaction between donor and acceptor occurs primarily via the GP excited by the donor at the surface of the GM [5].

In Fig. 2(right) we consider the ET rate, $k_{E T}$, as a function of the donor-acceptor separation, for the case when the donor position is kept fixed at $z_{D}=10 \mathrm{~nm}$ above the $\mathrm{GM}$, and the separation between the donoracceptor, $d=z_{D}-z_{A}$ is varied, for $\mu=1.0 \mathrm{eV}$ and $\mu=0.4 \mathrm{eV}$. At small separations we again use the Förster fitting presented earlier. To fit the behavior of the ET rate, $k_{E T}$, below the GM we choose the expression $f(z)=$ $A \exp (2 z / B)$, where the parameter $B$ will be connected with the penetration depth of the GP. The GM position is denoted by the dashed vertical line. We use the fitting function $f\left(z_{A}\right)$, and find for $\mathrm{B}$ the value $B=\delta_{S P}=$ $6.6 \mathrm{~nm}$, again showing that the main contribution to the ET rate comes from the GP on the GM. For the $\mu=$ $0.4 \mathrm{eV}$ case the ET rate, $k_{E T}$, is almost uninfluenced by the presence of the GM [5].

\section{CONCLUSION}

We investigated the behavior of quantum systems placed near a free standing graphene monolayer. We defined the ET efficiency and present its tunability for the varying values of the chemical potential. We saw that, for this specific pair of QEs, we can efficiently transfer energy over distances greater than300nm.

Furthermore we investigate the different mechanisms involved. We saw at small distances the Förster mechanism dominates, modified from its free space value due to the presence of the GM. For larger in-plane separations the main contribution is due to the propagating GP. When the $z$-separation between donor acceptor varied, the GP penetration depth dictates the interaction length.

\section{ACKNOWLEDGEMENT}

This work was supported by the Science Foundation Ireland under grant No. 10/IN.1/12975.

\section{REFERENCES}

[1] W. L. Barnes, A. Dereux, and T. W. Ebbesen, "Surface plasmon subwavelength optics," Nature, vol. 424, pp. 824-830, 2003.

[2] F. Javier Garcia de Abajo, "Graphene Plasmonics: Challenges and Opportunities," ACS Phot., vol. 1, pp. 135-152, 2014

[3] A. Yu. Nikitin, F. Guinea, F.J. Garcia-Vidal, and L. Martin-moreno, "Fields radiated by a nanoemitter in a graphene shett," Phys. Rev. B, vol. 84, 2011, 195446.

[4] H.T. Dung, L. Knöll, and D.-G. Welsch, "Intermolecular energy transfer in the presence of dispersing and absorbing media," Phys. Rev. A, vol. 65, 2002, 043813

[5] Vasilios D. Karanikolas, Cristian A. Marocico, and A. Louise Bradley, "Dynamical Tuning of Energy Transfer Efficiency on a Graphene Monolayer," accepted for Physical Review B 